\title{
Exploring Dynamic Expressions on Soft Wearables for Physical Exercises
}

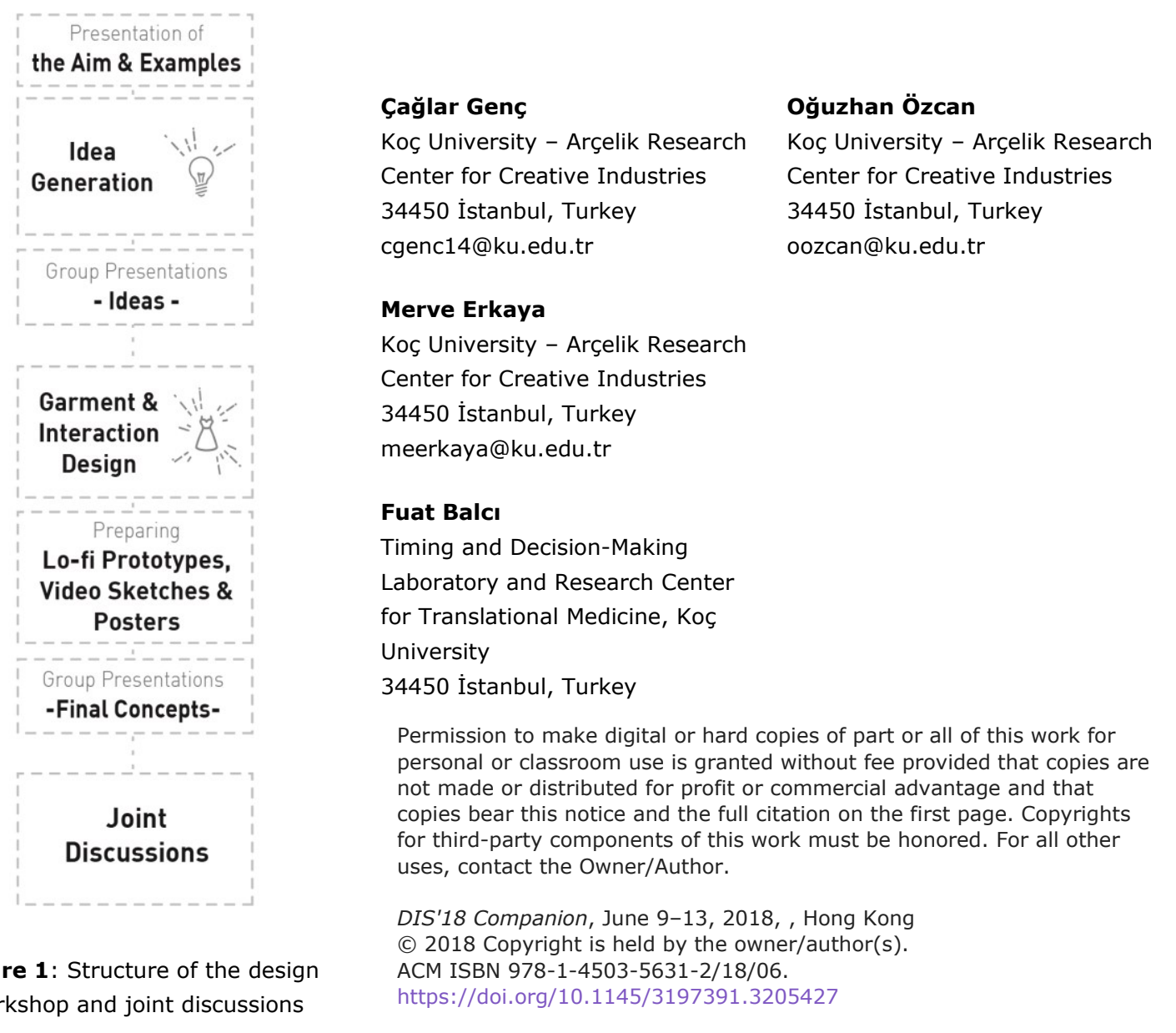

\begin{abstract}
Recent design approaches of wearables for physical exercising are often alienating the data from its specific experiences and/or limited to conventional display modalities for presenting information. As an alternative perspective, the aim of this paper is to explore in-situ social and individual experiences that activity related dynamic changes on garment surfaces might evoke in the context of exercising at the gyms. To investigate these, we conducted a design workshop ( $N=11,3$ gymgoers, 5 interaction and 3 fashion designers). Our results provide design insights for further research on how dynamic expression could alter (1) wearer's sense of achievement via solidifying unobservable efforts \& achievements and could (2) trigger social interactions.
\end{abstract}

\section{Author Keywords}

Soft Wearables, Sports Wearables, Fashion, Design Workshop.

\section{ACM Classification Keywords}

H.5.m. Information interfaces and presentation (e.g., $\mathrm{HCI}$ ): Miscellaneous.

\section{Introduction}

Wearable technologies for physical exercises have gained interest in HCI. Majority of the effort is to design wearable devices that utilize measurement and 
Figure 2: Group 1 (a) illustrations of garment alternatives, (b) a shot from video sketch
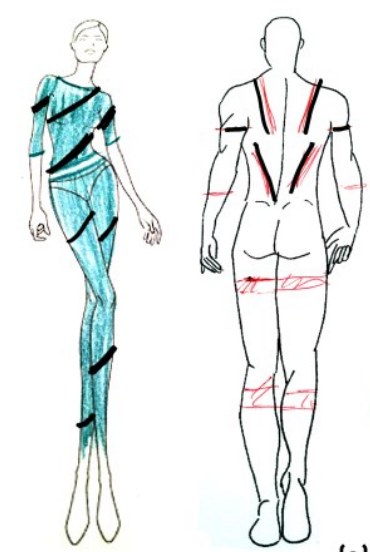

(a)

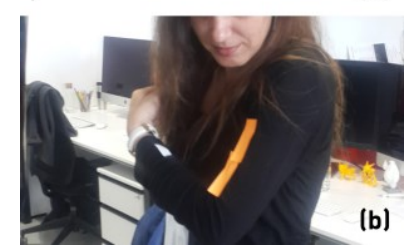

Group 1 explored a garment that dynamically expresses the progress of muscle building. The patterns, mapped to the muscle groups, start appearing as these muscles work. After appearing, the color of patterns turns from green to red to indicate progress. monitoring of the physical activity related performance to promote physical health. However, these devices often alienate the data from its experience. They monitor summary of performance following the exercise or providing complicated graphs about the performances of wearers trough smart watches or phones. Wearables that can contribute to the experiential aspects of actual exercising (i.e. feelings [15] or social interactions during exercises [10]) has been overlooked. This downscales the contribution of the wearables in real-life exercising experiences.

On the other hand, there are wearables that aim to support actual exercising experiences via constantly monitoring activity related information on wearable form factors $[12,14]$. Yet, these often rely on hard cases or interaction styles transferred from conventional displays. However, explorations should not be limited to hard cases or old interaction paradigms. The designers should also discover the meaning of soft and sartorial ways wearing and interacting with technology $[2,22]$.

Inspired by recent trend in HCI studies explored the merger between conventional materials (i.e. wood, textile) and computational technologies $[23,24]$, as an alternative, we propose focusing on dynamic alterations on the expressions of garment surfaces (i.e. pattern, color, silhouette changes [i.e. see 4,5,14]). To this end we aim to get insights on the physical activity related dynamic changes on soft wearables and how these could provide design opportunities for enhancing the individual and social experience of exercising. More specifically, we are interested in how such changes can be designed (1) to motivate in personal level, and (2) to influence social interaction during physical exercises.
We concentrated on the context of gym as an area of inquiry. Because (1) majority of people spend time in the gym for accomplishing physical activity [28], (2) the physical activities conducted in gyms provide rich sources of interactive opportunities related to these activities and (3) there are often multiple individuals exercising at the same time in gym, that social interactions are possible.

In this regard, we conducted a design workshop (see Figure 1), which included 3 potential users (i.e. people who regularly attend fitness activities), 3 fashion designers and 5 interaction designers $(\mathrm{N}=11)$. The workshop was followed by a joint discussion over projects. After analyzing the data from the workshop, we presented design insights for the further research on how dynamic expressions can complement individual and social exercising experiences.

\section{Related Work}

Majority of wearable technologies for physical exercises rely on detailed graphs, numbers on screens to monitor activity related information. These representations are irrelevant and too abstract for regular users to interpret [20]. Several studies took a different approach by aesthetically materializing the activity data in wearable forms through rapid fabrication technologies (i.e. see $[9,11,21])$. These artifacts provide personalized expressions about users' activity related achievements. Such expressions reflect back a sense of achievement and reward to the wearers. However, these systems rely on users' additional effort for producing these artifacts (i.e. via 3D printing) which might cause users to disengage in long-term use [21]. Moreover, the materialized artifacts are static in nature. How these 
Figure 3: Group 2 - an illustrative image of the soft wearable

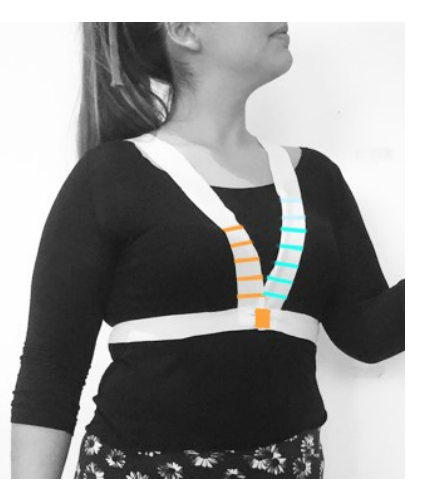

Group 2 designed a strapshaped soft wearable. The left strap indicates GYM attendance via horizontal lines emerging as wearer enters the GYM. With that, they aimed to foster social collaboration. They imagined the ones with more lines would be mentors to the others with less lines.

Moreover, the right strap is activated when wearer approaches to a GYM equipment. The data coming from the equipment will increase horizontal lines according to effort put on that specific physical activity. approaches might influence the actual physical exercising experiences is yet to be addressed.

In that direction, soft approaches to wearable designs often focuses on sensing technologies [i.e. 4,17]. However, several studies implemented situated wearable visualizations of activity during exercising experiences (i.e. shoe attached accessories with LEDs [12], 3D printed case with LEDs on arm to monitor over-training [3], screen-like LEDs implemented on shirt for social running [14], LEDs on helmets for social exertion [25]). The difference between those and our approach is we focus on opportunities soft wearables can present by augmenting clothing expressions (i.e. patterns). $[7,19]$ constitute as significant examples, in terms of designing soft wearable data representation, as well as aiming social experiences during physical activities. Yet, former examines the feedback on garments for learning outcomes of ballet training and the latter focus on in-game information on jerseys for creating awareness among basketball players.

\section{Design Workshop}

We reached to 11 participants, consisted of 3 potential users who, engage regular physical exercises at the gym, 3 fashion and 5 interaction designers. During the workshop (Figure 1), participants worked as groups. All groups had at least one of three kinds of audiences. Workshop lasted nine hours and yielded three concepts.

The workshop started with a brief presentation of our aim, which is generating ideas for the design of a soft wearable to be used during gym exercises. Then, we presented examples of commercial sport-wearables (i.e. Fitbit, Ralph Lauren's Polo Shirt), research prototypes (i.e. $[9,11,12])$ and dynamic expressions via computational composites (i.e. $[5,26])$. We limited our participants to design dynamic expressions related to physical activity on garments to be used at gyms. In the scope of this workshop, we were not concerned with the technological implementation of the concepts. Then, each group presented their concepts via posters and video sketches created with lo-fi prototypes [27]. The workshop followed by a discussion. Participants reflected on their decisions in terms of their potentials and challenges towards exercising experiences.

\section{Findings \& Discussions}

We, now report and discuss two themes that we derived from the presentations (see Figure 2-3-4) and the joint discussions. While the former contains insights for designing individual experiences, the latter focuses on the social experiences via dynamic expressions. In this section, due to limited space, we excluded some insights about instrumental uses (i.e. improving efficient training).

\section{SOLIDIFYING THE UNOBSERVABLE EFFORTS \& ACHIEVEMENTS ON BODY}

Participants stated their effort is limited in terms of the observability within one exercising session. As a reflection, all projects embraced the efforts and achievements as inputs for dynamic expressions. During discussion one interaction designer expressed their justification as "When you exercise for one day, your belly would not be thinner. Yet the dynamic expressions of your effort can reflect the idea "I really exercised" to yourself"

One gymgoer compared the dynamic expressions to the activity monitoring with small screens. Participants agreed that indications on larger areas of the body (i.e. 
Figure 4: Group 3 - (a) an illustrative image of garment (b) a shot of garment with red line indicating over training
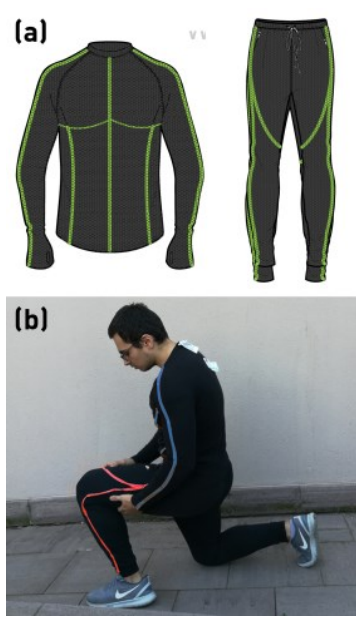

Group 3 imagined a garment which have shiny lines mapped with muscle groups. They expressed the wearer will stroke a muscle area to activate measuring. As the wearer works-out, the lines will turn color green to red in addition to that area gets slightly narrower to augment the feeling of the muscles working. The designers also meant to warn wearer with red lines as an indication of over training.
Figure 4), can act as a costume that might help them better identify the achievements with their selves comparing to dynamic changes on less visible part of the body (i.e. arm). They described the potential effect by referring to (what they call) superman effect. Superman is a regular guy in daily life, but when he wears the costume he feels and acts stronger. Such effect is also apparent in the empirical study of [1]. The researchers reported that participants who identified themselves with the symbolic meaning of a lab coat (presented as doctor's lab coat) and wore it has greater attentional abilities than the participants who identified themselves yet did not wear it.

Moreover, participats highlighted the symbollic meanings of body positions, where the dynamic expressions occur. For example, group 2 (Figure 3) expressed that they considered the chest area, since it is a surface used to express their achievement. They gave the example of medals on the chest for successes.

Also, all projects incorporated representations that indicate achievements level by level. For example, the more effort the wearer puts, the more lines appear (Figure 3). Participants foresaw that achieving a higher level might constitute a goal for the wearer. However, they highlighted these levels should be tailored to the capabilities or aims of the individual as there would differences between wearers.

Materialization of activity achievements in wearable form factors has been implemented and perceived as rewards [i.e. see 4, 5]. Different from them, our results suggest the dynamic alteration on the more visible and symbolically relevant areas of the body can help people to better identify themselves with the achievements during exercises. Moreover, leveled representations might be motivating within the exercise experience.

TRIGGERING SOCIAL INTERACTION AND COMPETITION One gymgoer expressed gym training as a lonesome experience that can be boring. Discussion yielded three ways that the dynamic expressions can overcome this lonely experience. First, seeing the increasing levels of achievements on others might trigger gamified competition during exercise. In case the wearers put too much effort that can harm their body, a warning mechanism about the over exertion was also proposed (i.e. Figure 4 - b). Second, the ones with higher achievements might be deemed as the ones to be consulted. Finally, people with similar level can be considered peers to exercise with. Although none of the projects implemented, one participant also highlighted that for the group activities (i.e. group cycling) the dynamic expressions can be increased through collaborative effort.

Although there are socio-motivational approaches commonly seen in previous attempts (i.e. through gamification [13], exertion games [16], collaborations [14]), our results suggested how dynamic pattern changes can trigger situated social interactions and competitions during the regular gym exercises.

\section{Conclusions \& Future Work}

The workshop and the discussions around workshop results helped us to explore the design insights on how dynamic alterations on soft wearables can complement individual and social in-situ experiences. Now, we plan to implement these insights into a research through design processes to detail the design space of the dynamic expressions in exercising scenarios. 


\section{References}

1. Hajo Adam and Adam D. Galinsky. 2012. Enclothed cognition. Journal of Experimental Social Psychology 48, 4: 918-925.

2. Joanna Berzowska. 2005. Electronic Textiles Wearable Computers, Reactive Fashion, and Soft Computation. Textile 3, 1: 58-75.

3. Marta Cecconi, Beatrice Baruzzo, Giovanni Caputo, and Rudy Faletra. 2014. NAERO light your energy. Proceedings of the 2014 ACM International Symposium on Wearable Computers Adjunct Program - ISWC '14 Adjunct: 33-38.

4. L. Ponce Cuspinera, Sakura Uetsuji, F. J. Ordonez Morales, and Daniel Roggen. 2016. Beach volleyball serve type recognition. Proceedings of the 2016 ACM International Symposium on Wearable Computers - ISWC '16 fig 3: 44-45.

5. Laura Devendorf, Joanne Lo, Noura Howell, et al. 2016. "I don't want to wear a screen": Probing perceptions of and possibilities for dynamic displays on clothing. Proc. CHI '16, ACM, 60286039.

6. Çağlar Genç, Oğuz Turan Buruk, Oğuzhan Özcan, Sejda Inal Yilmaz, and Kemal Can. 2017. Forming Visual Expressions With Augmented Fashion. Visual Communication 16, 4: 427-440.

7. James Hallam, Alison McKenna, Emily Keen, Mudit Gupta, and Christa Lee. 2014. Ballet hero. Proceedings of the 2014 ACM International Symposium on Wearable Computers Adjunct Program - ISWC '14 Adjunct: 49-54.

8. Margot Jacobs and Linda Worbin. 2005. Reach: dynamic textile patterns for communication and social expression. $\mathrm{CHI}$ '05 extended abstracts: 1493-1496.

9. Rohit Ashok Khot, Larissa Hjorth, and Florian "Floyd" Mueller. 2014. Understanding physical activity through 3D printed material artifacts. Proc. CHI '14: 3835-3844.

10. Kristina Knaving, Paweł Wołniak, Morten Fjeld, and Staffan Björk. 2015. Flow is Not Enough:
Understanding the Needs of Advanced Amateur Runners to Design Motivation Technology. $\mathrm{CHI}$ 2015, Crossings: 2013-2022.

11. Moon-hwan Lee, Seijin Cha, and Tek-jin Nam. 2015. Patina Engraver: Visualizing Activity Logs as Patina in Fashionable Trackers. Proc. CHI '15, ACM 1173-1182.

12. Brian Y Lim, Aubrey Shick, Chris Harrison, and Scott Hudson. 2011. Pediluma: Motivating Physica Activity Through Contextual Information and Social Influence. Tei 2011: 173-180.

13. James J. Lin, Lena Mamykina, Silvia Lindtner, Gregory Delajoux, and Henry B. Strub. 2006. Fish'n'Steps: Encouraging Physical Activity with an Interactive Computer Game. 261-278.

14. M Mauriello, Michael Gubbels, and Je Froehlich. 2014. Social Fabric Fitness: The Design and Evaluation of Wearable E-Textile Displays to Support Group Running. Chi '14: 2833-42.

15. Florian "Floyd" Mueller and Damon Young. 2017. Five Lenses for Designing Exertion Experiences. Proc. CHI '17: 2473-2487.

16. Florian Mueller and Katherine Isbister. 2014 Movement-based game guidelines. Proc. CHI '14: 2191-2200.

17. Le T. Nguyen, Ming Zeng, Patrick Tague, and Joy Zhang. 2015. Recognizing new activities with limited training data. Proceedings of the 2015 ACM International Symposium on Wearable Computers ISWC '15: 67-74.

18. Linnéa Nilsson, Mika Satomi, Anna Vallgårda, and Linda Worbin. 2011. Understanding the complexity of designing dynamic textile patterns. Ambience.

19. Mitchell Page and Andrew Vande Moere. 2007 Evaluating a Wearable Display Jersey for Augmenting Team Sports Awareness. Proceedings of the 5th International Conference on Pervasive Computing 4480: 91-108.

20. Jill Walker Rettberg. 2014. Seeing ourselves through technology: How we use selfies, blogs and 
wearable devices to see and shape ourselves. Springer.

21. Simon Stusak, Aurelien Tabard, Franziska Sauka, Rohit Ashok Khot, and Andreas Butz. 2014. Activity sculptures: Exploring the impact of physical visualizations on running activity. IEEE

Transactions on Visualization and Computer Graphics 20, 12: 2201-2210.

22. Oscar Tomico, Lars Hallnäs, Rung-huei Liang, and Stephan A G Wensveen. 2017. Towards a Next Wave of Wearable and Fashionable Interactions. 11, 3: 1-6.

23. Anna Vallgårda. 2014. Giving form to

computational things: Developing a practice of interaction design. Personal and Ubiquitous Computing 18, 3: 577-592.

24. Anna Vallgårda and Tomas Sokoler. 2010. A material strategy: Exploring material properties of computers. International Journal of Design 4, 3: 114

25. Wouter Walmink, Danielle Wilde, and Florian "Floyd" Mueller. 2013. Displaying heart rate data on a bicycle helmet to support social exertion experiences. Proc. TEI '14 February: 97-104.

26. Lining Yao, Jifei Ou, Chin-Yi Cheng, et al. 2015. bioLogic: Natto Cells as Nanoactuators for Shape Changing Interfaces. Proc. CHI '15: 1-10.

27. John Zimmerman. 2005. Video Sketches: Exploring pervasive computing interaction designs. IEEE Pervasive Computing 4, 4: 91-94.

28. Fitness Industry Analysis 2018 - Cost \& Trends. Retrieved from

https://www.franchisehelp.com/industryreports/fitness-industry-analysis-2018-costtrends/. 